\title{
Involvement of Glycogen in Morphogenesis of Coprinus cinereus
}

\author{
By RAIDA I. JIRJIS AND D. MOORE \\ Department of Botany, The University, Manchester $M_{13} 9 P L$
}

(Received I3 November 1975)

\begin{abstract}
SUMMARY
The quantity of glycogen that could be extracted from cultured dikaryotic mycelia of Coprinus cinereus increased during the first 5 days of growth but subsequently declined. The decline coincided with the main phase of removal of carbohydrate from the culture medium, with a major reduction in the level of soluble reducing sugars of the mycelium, and with the appearance of mature sclerotia. This result complements earlier electron microscope observations that intracellular accumulations of glycogen increase during sclerotium formation and decrease during sclerotium maturation. However, extractable glycogen reserves also accumulated and then dispersed under culture conditions which did not allow sclerotium formation and in strains unable to form sclerotia. It is concluded that glycogen also serves as a transient carbohydrate store for aspects of vegetative mycelial morphogenesis other than sclerotium production.
\end{abstract}

\section{INTRODUCTION}

Cytochemical observations have repeatedly implicated a glycogen-like polysaccharide in the development of the basidiomycete Coprinus cinereus ( $=C$. lagopus). After only 2 days growth, hyphal cells of the dikaryotic mycelium swell and accumulate large amounts of glycogen, the stored polysaccharide being mobilized and translocated to sporophores that are formed later (Madelin, 1960). The earliest stages in sporophore development involve the formation of a spherical mass of cells, and in many of the cells of this sporophore initial, the glycogen reserves are re-established. These glycogen accumulations remain at the base of the stipe of the developing fruit body (Matthews \& Niederpruem, 1973). At later stages, glycogen becomes concentrated in distinct zones in the hymenium of the gills of expanding fruits and is translocated into the spores when sporulation occurs (Bonner et al., 1957). In the vegetative mycelium, glycogen is involved in the maturation of small perennating bodies called sclerotia (Waters, Moore \& Butler, $1975 b$ ). These are also initiated as a spherical mass of hyphal cells, and the beginning of cellular differentiation is marked by the accumulation of glycogen. However, the accumulated glycogen appears to be utilized as maturation proceeds, the decline in the amount of intracellular glycogen being matched by concurrent synthesis of a thick secondary cell wall of unusual structure (Waters, Butler \& Moore, 1972). In all of these studies, the identification of the polysaccharide rests on light- and/or electronmicroscope observations. The work reported here was undertaken to provide further information on the identity of the polysaccharide as well as a chemical assessment of its concentration in the mycelium during growth and sclerotium formation. 


\section{METHODS}

Organism and culture. The organism used was Coprinus cinereus (Schaeff. ex Fr.) S. F. Gray sensu Konr. (= C. lagopus sensu Lewis). Most work was done on a dikaryotic mycelium (code number RAI) constructed from the wild-type monokaryons BC9/6,6 and ZBw60I/40,40. Other strains have been described previously (Waters et al., 1975b). All cultures were grown at $37^{\circ} \mathrm{C}$ unless stated otherwise. For routine purposes, maltose-CM (Stewait \& Moore, 1974) was the usual medium. In a few experiments, the yeast extract-malt extract-glucose (YMG) medium described by Gooday (1974) was used, but in most cases a fully defined basal medium (the SNC medium of Stewart \& Moore, 1974) was used. The SNC medium contained Io mM- $\mathrm{Na}_{2} \mathrm{HPO}_{4}$, IO mM- $\mathrm{KH}_{2} \mathrm{PO}_{4}$, and $3 \mu \mathrm{M}$-thiamin hydrochloride: it was made up with tap water and solidified, if necessary, with $\mathrm{I} \cdot 5 \%(\mathrm{w} / \mathrm{v})$ Difco Bacto-agar. After addition of the appropriate nitrogen sources (usually $25 \mathrm{~mm}$-ammonium tartrate), the medium was autoclaved for $15 \mathrm{~min}$ at $\mathrm{I} 2 \mathrm{I}{ }^{\circ} \mathrm{C}$. Solutions of carbon sources (usually maltose, to give a final concentration of $25 \mathrm{~mm}$ ) were autoclaved separately, and mixed with the rest of the medium when both solutions had cooled to about $50^{\circ} \mathrm{C}$.

Chemical analysis. Samples of the medium were analysed for ammonium nitrogen (McCullough, 1967), amino nitrogen (Lewis, 1963) and reducing sugars (Somogyi, 1952). Samples of the mycelium were treated three times, without grinding, with ethanol (approx. $5 \mathrm{ml} / 500 \mathrm{mg}$ fresh wt) at $30{ }^{\circ} \mathrm{C}$ for $20 \mathrm{~min}$. The residue was collected each time by centrifuging and, after a final wash with $10 \mathrm{ml}$ water, was used for the determination of mycelial protein (Lowry et al., 195I). The supernatants were combined with the wash water and used for estimation of ammonia, amino nitrogen and soluble reducing sugar. Separate mycelium samples were used to determine total nitrogen by the Kjeldahl method and total carbohydrate with the anthrone reagent. Glycogen was extracted by the method of Hassid \& Abraham (I957) from mycelium (approx. I00 mg fresh wt) that had been frozen with liquid nitrogen and ground with acid-washed sand. The glycogen was measured as described by Krisman (1962), commercial rabbit liver glycogen being used as a standard.

Measurement of sclerotia. Sclerotium production was quantified by counting with the aid of a dissecting microscope. Immature sclerotia (white tufts of aerial hyphae) and mature sclerotia (brown-black compact spheroids) were enumerated separately in 25 to 30 fields of view scattered across two orthogonal diameters of the culture. About $35 \%$ of the total surface area of the culture was examined in this way. Three replicates for each treatment were scored and all counts contributed to the final measure of 'sclerotia per colony'.

\section{RESULTS AND DISCUSSION}

The polysaccharide extracted from Coprinus formed an iodine complex having an absorption spectrum very similar to that of authentic glycogen. The latter had a fairly broad absorption peak with a maximum at $474 \mathrm{~nm}$. The Coprinus material also had a broad peak but with a maximum at $467 \mathrm{~nm}$. This suggests a close similarity in structure between the two polysaccharides and provides justification for our referring to the Coprinus polysaccharide as glycogen.

The accumulation of mycelial glycogen reached a maximum about 5 days after the start of incubation in stationary cultures (Fig. I). The amount of glycogen declined as incubation continued and as sclerotia were produced. Waters et al. (1975b) compared the numbers of electron microscopically visible glycogen particles with the wall thickness of sclerotial medullary cells. They observed a decline in the number of glycogen particles and considered 


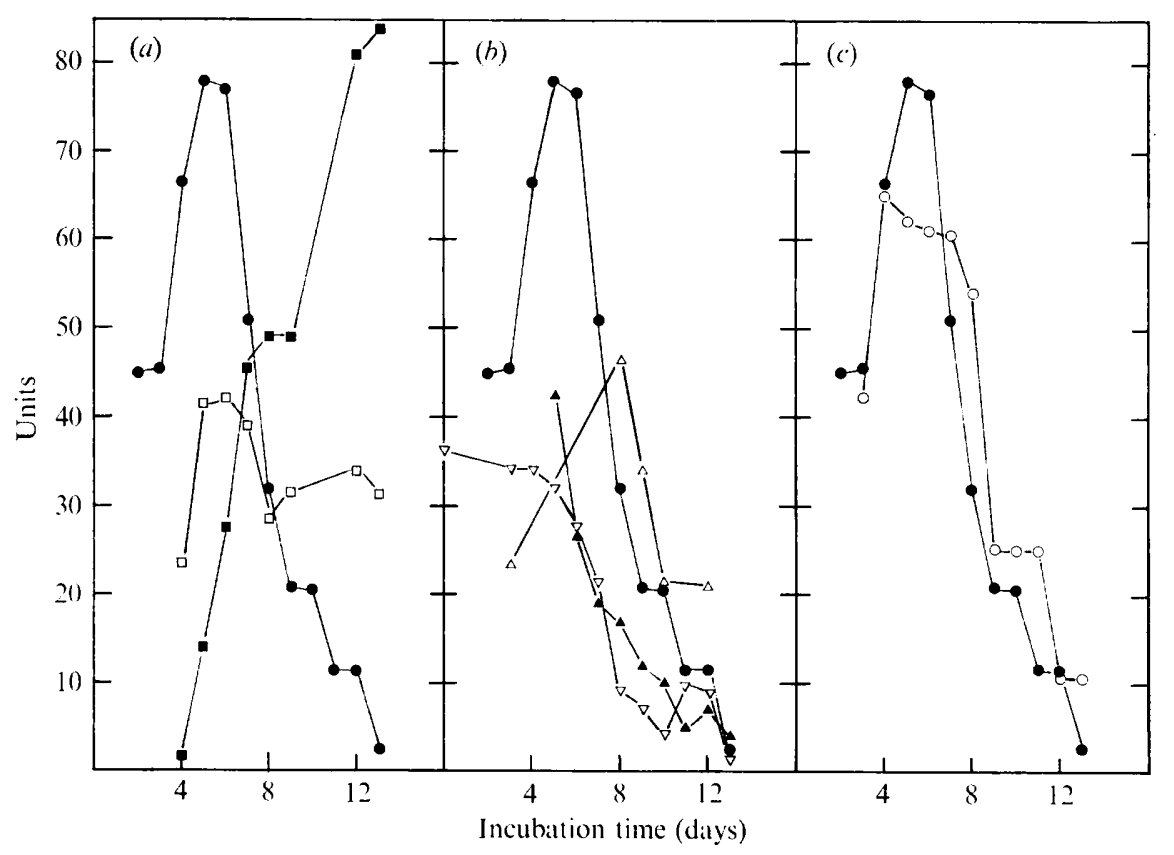

Fig. I. Comparison of glycogen accumulation by mycelium grown in stationary culture with (a) sclerotium production and maturation, $(b)$ changes in the carbohydrate levels in the medium and mycelium, and (c) glycogen accumulation by similar mycelia grown in shake culture. $\bigcirc$, Glycogen in mycelia from stationary cultures (mg glycogen/g dry wt); $\mathbf{\square}$, mature sclerotia and $\square$, immature sclerotia, both expressed in thousands per colony; $\boldsymbol{\Delta}$, soluble reducing sugars in the mycelium (mg carbon/g dry wt); $\triangle$, total mycelial carbohydrate (mg carbon/Ioo mg dry wt); $\nabla$, concentration of reducing sugars in the medium ( $\mathrm{mg}$ carbon/10 $\mathrm{ml}) ; 0$, glycogen in mycelia from shake cultures (mg glycogen/g dry wt).

this to be the result of a real reduction in the glycogen concentration and not just a redistribution of the polysaccharide. Our data support this view. The glycogen concentration of the colony as a whole is substantially reduced as the sclerotia mature. Clearly, the polysaccharide is utilized and not simply redistributed; and in view of the ultrastructural observations it seems likely that the glycogen is converted into the material which is used to construct the secondary wall of medullary cells.

The decline in glycogen content of the colony coincides with a reduction in the level of soluble reducing sugars of the mycelium and also with the major phase of utilization of carbohydrate from the medium (Fig. I). Total mycelial carbohydrate reached a maximum 3 days after the peak in the glycogen curve, and subsequently declined, although at a much slower rate. The turning point in most of these curves occurred after about 5 days incubation. By this time the colony was sufficiently well established to cover the medium completely: thus, the most dramatic changes in carbohydrate levels in both medium and mycelium occurred after cessation of invasive growth and seem to be related to colony maturation rather than to colony formation. Furthermore, only carbohydrates showed these rapid fluxes. Total lipid levels remain essentially constant during growth of the colony (Darbyshire, 1974), while our own analyses show that concentrations of mycelial protein, total nitrogen and ammonium nitrogen fluctuated during 16 days incubation but without any clear trend. A colony grown in a crystallizing dish containing $100 \mathrm{ml}$ of defined medium used approximately half of the nitrogen in the medium in 16 days, the rate of removal being fairly con- 
Table I. Concentrations of glycogen in mycelia of a variety of wild-type strains during growth in liquid medium in stationary culture

The medium used was SNC +25 mM-ammonium tartrate +25 mM-maltose. Results are expressed as $\mathrm{mg}$ glycogen $\mathrm{g} / \mathrm{dry}$ wt mycelium.

\begin{tabular}{|c|c|c|c|c|c|c|}
\hline \multirow[t]{2}{*}{ Identity of mycelium } & \multicolumn{6}{|c|}{$\begin{array}{l}\text { Glycogen concentration after incubation for } \\
\text { (days): }\end{array}$} \\
\hline & 3 & 5 & 7 & 9 & I I & 13 \\
\hline zBw60I/40,40 (monokaryon) & $22 I$ & 263 & $22 I$ & 201 & 102 & 95 \\
\hline RAI (dikaryon) & 46 & 78 & 51 & 20 & 12 & 3 \\
\hline G2 (monokaryon) & 56 & 65 & 54 & $6 I$ & 55 & 40 \\
\hline HI (monokaryon) & 84 & 84 & 63 & 42 & 56 & 28 \\
\hline вс9/6,6 (scl-r)* & 88 & 58 & 32 & $2 I$ & 12 & 9 \\
\hline H9 (scl-I) & 37 & 26 & II & 12 & 10 & 8 \\
\hline BI $(s c l-2)$ & 74 & 72 & 81 & 87 & 58 & 9 \\
\hline $2 \mathrm{HI}(s c l-3)$ & 2 I I & 133 & 123 & 98 & 47 & 28 \\
\hline LI $(s c l-4)$ & 100 & 137 & 132 & 120 & I I 4 & 56 \\
\hline
\end{tabular}

* All $s c l$ strains are monokaryons that are unable to make sclerotia because of a recessive genetic defect.

stant over the whole period. This corresponds to the utilization of about $\mathrm{I} \cdot 5 \mathrm{mmol}$ ammonium by a colony with an average dry weight of $150 \mathrm{mg}$. In contrast, when such a colony was grown on a complex medium with a very low initial ammonium concentration (maltose-CM or YMG), it excreted about the same quantity of ammonia into the medium when producing sclerotia. Colonies producing sporophores have also been observed to excrete ammonia into the medium (Stewart \& Moore, I974).

Clearly, glycogen is accumulated in young mycelium and is utilized as the sclerotia mature. However, it is probably also used as a transient carbohydrate storage material in a number of different aspects of vegetative differentiation in much the same way as it is involved in different stages of sporophore development. Sclerotia were not produced by mycelia grown in shake culture, yet a transient accumulation of glycogen was still observed. The maximum level was only 75 to $80 \%$ of that reached by stationary cultures, was attained earlier, and was maintained for a longer period, but the glycogen was eventually utilized (Fig. I). Furthermore, analysis of a range of different wild-type strains grown under conditions which favoured sclerotium production showed a considerable variation in the amount of glycogen accumulated (Table I). Even $\mathrm{scl}$ strains, which are genetically incapable of making mature sclerotia (Waters et al., $1975 b$ ), were able to accumulate large quantities of glycogen during the first week of growth and subsequently utilize it.

We conclude that although a glycogen synthesis-utilization cycle undoubtedly plays a part in sclerotium maturation, this developmental pathway is only one of a number that are open for glycogen utilization. Gross chemical analysis cannot distinguish between glycogen accumulations stored for different purposes, so histological methods are still required to differentiate the alternatives. It is worth noting, however, that in terms of cellular differentiation, the alternatives may not be very different. Maturation of the colony involves largescale differentiation of the submerged mycelium (Waters, Butler \& Moore, I975a) which produces cell types virtually identical to those found in sclerotia. Consequently if sclerotium formation is not possible, the same sorts of cells may be produced in a different tissue and this may require a glycogen synthesis-utilization cycle of the same type as that which characterizes sclerotium maturation. 


\section{REFERENCES}

Bonner, J. T., Hoffman, A. A., MoriokA, W. T. \& Chiquoine, A. D. (I957). Distribution of polysaccharides and basophilic substances during the development of the mushroom Coprinus. Biological Bulletin. Marine Biological Laboratory, Woods Hole, Mass. I12, I-6.

Darbyshire, J. (1974). Developmental studies on Coprinus lagopus. Ph.D. thesis, University of Manchester.

Gooday, G. W. (1974). Control of development of excised fruit bodies and stipes of Coprinus cinereus. Transactions of the British Mycological Society 62, 39I-399.

HASSID, W. Z. \& ABRAHAM, S. (1957). Chemical procedures for the analysis of polysaccharides. Methods in Enzymology, vol. 3, pp. 34-50. Edited by S. P. Colowick and N. O. Kaplan. New York: Academic.

Krisman, C. R. (1962). A method for the colorimetric estimation of glycogen with iodine. Analytical Biochemistry 4, 1 7-23.

LEWIS, D. K. (I963). A study of amino acids and their metabolism in Torulopsis utilis. Ph.D. thesis, University of Bristol.

Lowry, O. H., Rosebrough, N. J., Farr, A. L. \& Randall, R. J. (I95I). Protein measurement with the Folin phenol reagent. Journal of Biological Chemistry r93, 265-275.

McCullough, A. (1967). The determination of ammonia in whole blood by a direct colorimetric method. Clinica chimica acta $\mathbf{1 7}, 297$.

Madelin, M. F. (1960). Visible changes in the vegetative mycelium of Coprinus lagopus Fr. at the time of fruiting. Transactions of the British Mycological Society 43, 105-1 10.

MAtThews, T. R. \& NiederPruem, D. J. (1973). Differentiation in Coprinus lagopus. II. Histology and ultrastructural aspects of developing primordia. Archiv für Mikrobiologie 88, 169-1 80.

SoMOGYI, M. (1952). Notes on sugar determination. Journal of Biological Chemistry r95, 19-23.

STEWART, G. R. \& MOORE, D. (1974). The activities of glutamate dehydrogenases during mycelial growth and sporophore development in Coprinus lagopus (sensu Lewis). Journal of General Microbiology 83, 73-81.

WAters, H., Butler, R. D. \& Moore, D. (1972). Thick-walled sclerotial medullary cells in Coprinus lagopus. Transactions of the British Mycological Society 59, 167-169.

WATERs, H., Butler, R. D. \& MOORE, D. (1975a). Structure of aerial and submerged sclerotia of Coprinus lagopus. New Phytologist 74, 199-205.

WATERS, H., MOORE, D. \& BUTLER, R. D. (I975b). Morphogenesis of aerial sclerotia of Coprinus lagopus. New Phytologist 74, 207-213. 